\title{
Long-term outcomes of the frozen elephant trunk procedure: a systematic review
}

\author{
David H. Tian ${ }^{1,2}$, Hakeem Ha ${ }^{1}$, Yashutosh Joshi ${ }^{1}$, Tristan D. Yan ${ }^{1,3}$ \\ ${ }^{1}$ Collaborative Research (CORE) Group, Macquarie University, Sydney, Australia; ${ }^{2}$ Department of Anaesthesia and Perioperative Medicine, \\ Westmead Hospital, Sydney, Australia; ${ }^{3}$ Department of Cardiothoracic Surgery, Royal Prince Alfred Hospital, Sydney, Australia \\ Correspondence to: Dr. David H. Tian. Collaborative Research (CORE) Group, Macquarie University, Balaclava Rd, Macquarie Park, NSW 2109, \\ Australia. Email: drdavidtian@gmail.com.
}

\begin{abstract}
Background: The frozen elephant trunk (FET) procedure remains an increasingly popular approach to address complex multi-segmental aortic pathologies, owing to their ability to promote false lumen thrombosis and reduce the need for second-stage operations. While the short-term outcomes of such procedures have been shown to be acceptable, much less is known regarding long-term outcomes. This systematic review evaluates long-term outcomes of the FET procedure.

Methods: Studies with at least 12 months follow-up data on FETs were identified in four electronic databases. All studies were reviewed by two independent researchers and relevant data extracted. Long-term outcomes, including overall survival, freedom from reintervention, and freedom from aortic events, were evaluated using patient data recreated from digitized Kaplan-Meier curves.

Results: Thirty-seven studies with 4,178 patients were identified. The majority of the studies focused solely on acute dissections. Average follow-up was 3.2 years. Overall survival at 1-, 3-, and 5-year was 89.6\%, $85.2 \%$, and $82.0 \%$, respectively. Freedom from reintervention at the same timepoints were $93.9 \%, 89.3 \%$, and $86.8 \%$, respectively. Mortality, permanent neurological deficit and spinal cord injury were $10.2 \%, 7.7 \%$, and $6.5 \%$, respectively.

Conclusions: Survival after the FET procedure is favorable, though ongoing close serial monitoring is essential to assess for the need for further reintervention. Larger multi-institutional registries are required to provide more robust evidence to better elucidate the patient cohort that would most benefit from the FET.
\end{abstract}

Keywords: Frozen elephant trunk (FET); stent-graft; survival; freedom from reintervention; systematic review

Submitted Feb 03, 2020. Accepted for publication Feb 24, 2020.

doi: $10.21037 /$ acs.2020.03.08

View this article at: http://dx.doi.org/10.21037/acs.2020.03.08

\section{Introduction}

Combining the advantages of the classical elephant trunk with modern stent technology, the frozen elephant trunk (FET) procedure has been instrumental in treating complex multi-segmental aortic pathologies in a single operation. The secured expansile stent-graft is able to facilitate downstream aortic remodelling by inducing false lumen thrombosis and depressurization of the false lumen, stabilize the dissecting membrane, and limit stent-graft migration and proximal type Ia endoleaks $(1,2)$. While multiple metaanalyses have reaffirmed the relatively safe short-term profiles of these devices, much less is known regarding long-term outcomes, particularly in terms of overall survival and freedom from reintervention (3-7). The present metaanalysis aimed to determine long-term outcomes following the FET procedure.

\section{Methods}

\section{Literature search strategy}

Electronic searches were performed using Ovid Medline, Embase, Scopus, and PubMed, from their date of inception 
to October 2019. To achieve maximum sensitivity of the search strategy, the terms 'elephant trunk', 'Thoraflex', 'E-vita', 'Gianturco Z', 'Chavan-Haverich', or 'Cronus' were used as either keywords or MeSH terms. Determination of whether the descending endoprosthesis was stented (i.e., 'frozen') or not (i.e., conventional elephant trunk) was made upon full article review. The reference lists of all included studies were reviewed for further identification of other potentially relevant studies. All identified articles were systematically assessed using the inclusion and exclusion criteria.

\section{Selection criteria}

Eligible studies for the present systematic review included those which (I) examined the use of FETs, (II) had clinical follow-up data of at least 12 months, and (III) had at least 10 patients. The FET is required to be deployed via open surgery in an antegrade fashion into the proximal descending aorta, and secured at the proximal aspect by sutures. No distinction was made regarding the management of head and neck vessels. All publications were limited to those involving human subjects and in the English language. Abstracts, case reports, conference presentations, editorials, and expert opinions were excluded. Review articles were omitted because of potential publication bias and duplication of results. Primary endpoint was overall survival. Secondary outcomes included freedom from reintervention, freedom from aortic events, 30 day/inhospital mortality, stroke/permanent neurological damage, spinal cord damage, temporary neurological deficit, acute kidney injury, and hospital and intensive care unit (ICU) length of stay.

\section{Data extraction and critical appraisal}

All data were extracted from article texts, tables and figures. Two investigators (Y.J., H.H.) independently reviewed each retrieved article. Discrepancies between the two reviewers were resolved by the senior investigator (D.H.T.). Quality assessment was assessed using a modified schema used for assessing case series, developed by the Institute of Health Economics (Alberta, Canada) (8) (Table S1). This schema examines the suitability of study objective, design, population, intervention, outcome measure, statistical analysis, appropriateness of results and conclusions, and competing interests (Table S1). Each study was scored out of 15 points, with
13-15 representing high-quality, $10-12$ as medium-quality, and less than 10 as low-quality.

\section{Statistical analysis}

Descriptive statistics were calculated for all collected variables. Categorical or continuous variables were aggregated using meta-analysis of proportions or means, as appropriate. Data is presented as $\mathrm{N}(\%)$ or mean \pm standard deviation (SD). Where continuous values are presented in median with range or interquartile ranges they were converted to mean and SD using methods published by Wan and colleagues (9). Guyot's iterative algorithm was applied to digitized Kaplan-Meier curves to reconstruct individual patient data $(10,11)$. This approach assumed a constant, non-informative censoring mechanism. The reconstructed patient data were then aggregated to form the combined survival curve. The estimated survival for a 57-year-old male in 2010, representing the median age, sex, and study period of all studies, is also plotted to represent general population survival curve. The American life tables were selected arbitrarily (Center for Disease Control, United States). All p-values were two-sided, and p-values less than 0.05 were considered statistically significant. All statistics were performed with $\mathrm{R}$ (version 3.3.5, R Core Team, Vienna, Austria).

\section{Results}

\section{Literature search}

Overall 2,084 records were identified from the literature search (Figure S1). Following review (1,2,12-29), 37 were included in the quantitative analysis with a total of 4,178 patients (Table S2) (30-46). No further studies were identified from review of references. Three studies were multi-center studies $(12,14,42)$, including an international registry (14). The median size of included studies was 58 patients (interquartile range, 34-120). Most studies were published by Chinese centers (12 studies), followed by German (8 studies) and Japanese centers (7 studies). Median duration of study was 7 years, with average follow-up of 3.2 years.

\section{Patient characteristics}

FETs were used exclusively for acute dissections in 23 studies involving 1,801 patients. In 10 studies the patient 


\begin{tabular}{|c|c|c|c|c|c|}
\hline Male (\%) & 72 [68-75] & $73[68-78]$ & 76 [68-83] & $68[61-75]$ & $3,415(24)$ \\
\hline Renal dysfunction (\%) & $8[6-11]$ & $7[5-11]$ & $11[6-19]$ & 9 [4-18] & $3,249(24)$ \\
\hline Concomitant CABG (\%) & $11[9-14]$ & $10[8-12]$ & $14[8-21]$ & 13 [9-19] & $3,324(29)$ \\
\hline CPB time (mins) & 206 [191-220] & 199 [177-221] & 202 [179-225] & 222 [200-243] & $3,568(32)$ \\
\hline Lowest temperature $\left({ }^{\circ} \mathrm{C}\right)$ & 23 [23-24] & 24 [24-24] & 23 [23-24] & 26 [25-26] & $3,224(29)$ \\
\hline
\end{tabular}

Data is presented as value [95\% confidence interval]. ACP, antegrade cerebral perfusion; CABG, coronary artery bypass grafting; CPB, cardiopulmonary bypass; HCA, hypothermic circulatory arrest.

cohorts were chronic dissections or elective surgeries (698 patients). In the remaining studies there was a mixture of emergent and elective indications. A variety of stent-grafts were used, including E-Vita Open/E-Vita Open Plus (13 studies), Cronus (10 studies), Thoraflex (6 studies), GORE TAG (3 studies), Valiant (2 studies), Medtronic TX2 (2 studies), JSOG (2 studies), as well as Frozenix (1 study), Gianturco stent/Hemashield Gold graft (1 study), and Chavan-Haverich (1 study).

Average age of included patients was 57 years old (IQR, 54-60 years), with $72 \%$ males (Tables 1,S3). The majority of patients were hypertensive $(76 \%)$, with a small proportion having diabetes $(8 \%)$, and renal dysfunction $(8 \%)$. Other comorbidities, such as respiratory dysfunction, Marfan's syndrome, previous surgery, were insufficiently reported. Average cardiopulmonary bypass and cross-clamp times were 206 minutes and 118 minutes, respectively (Table S4). Average hypothermic circulatory arrest time was 46 minutes with antegrade cerebral perfusion time of 63 minutes (where reported). Circulatory arrest occurred at $23{ }^{\circ} \mathrm{C}$ on average.

\section{Overall survival}

Overall survival at 1-, 2-, 3-, 5-, and 10-year were $89.6 \%, 87.1 \%, 85.2 \%, 82.0 \%$, and $68.0 \%$, respectively (Figure 1). Survival at 1-, 2-, 3-, 5-, and 10-year for studies that reported only acute dissections were $90.7 \%, 88.3 \%$, $86.1 \%, 83.9 \%$, and $73.5 \%$, compared to $90.0 \%, 87.4 \%$, $85.2 \%, 79.1 \%$, and $56.0 \%$ for studies that only included chronic dissections/elective aneurysmal patients. Freedom from reintervention at 1-, 2-, 3-, and 5-year were 93.9\%, $91.6 \%, 89.3 \%$, and $86.8 \%$, respectively (Figure 2 ). Freedom from aortic events at 1-, 2-, 3-, and 5-year were $98.3 \%$, $96.2 \%, 91.3 \%$, and $86.6 \%$, respectively (Figure 3).

\section{Secondary outcomes}

Pooled in-hospital/30-day mortality was $10.2 \%$ (Tables 2,S5). Permanent neurological deficit and spinal cord injury were $7.7 \%$ and $6.5 \%$, respectively. Acute kidney injury, with varying definitions, was $15.5 \%$. There were insufficient data to evaluate temporary neurological deficit and hospital and ICU length of stay.

\section{Quality assessment}

The majority of studies were assessed to be medium-quality, with one high-quality and seven low-quality studies. Almost all of the studies were retrospective, single center trials, with no predetermined definitions of clinical outcomes. Loss to follow-up and the consecutive nature of patient enrolment were also inconsistently reported. 


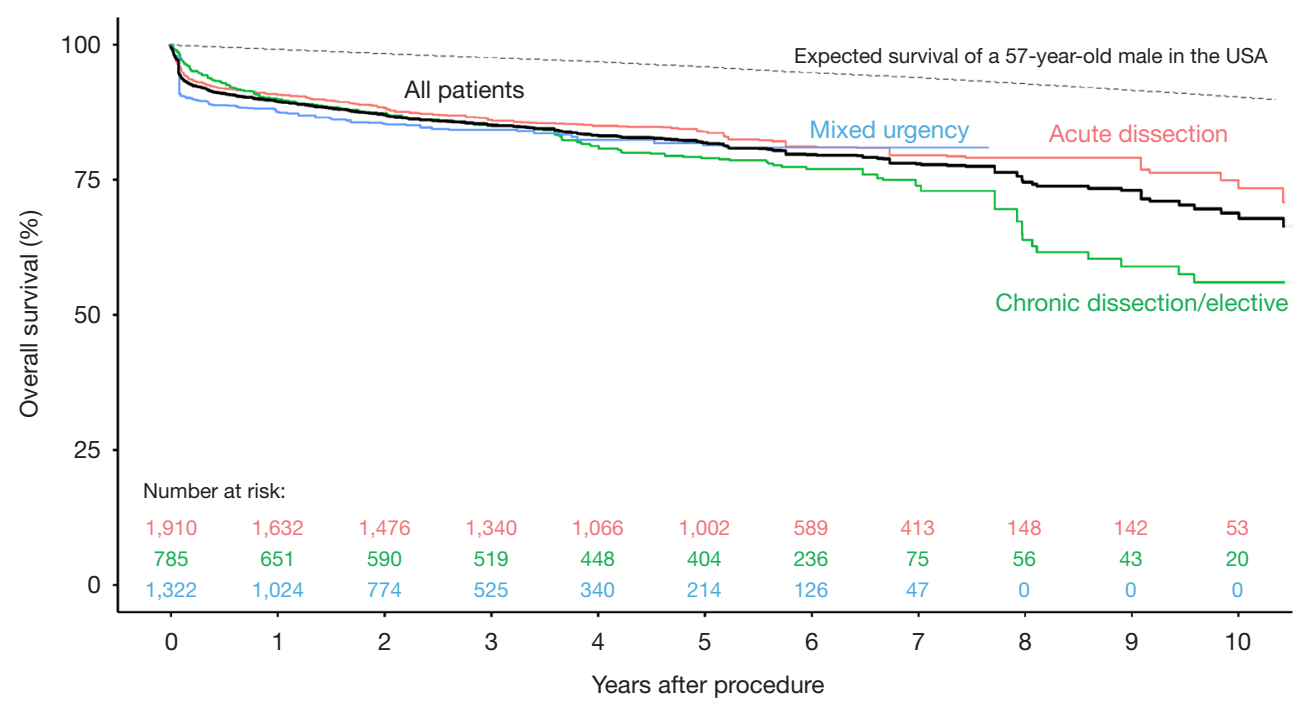

Figure 1 Overall survival. Dotted line represent expected survival of the general population, using the study mean age/gender as a reference.

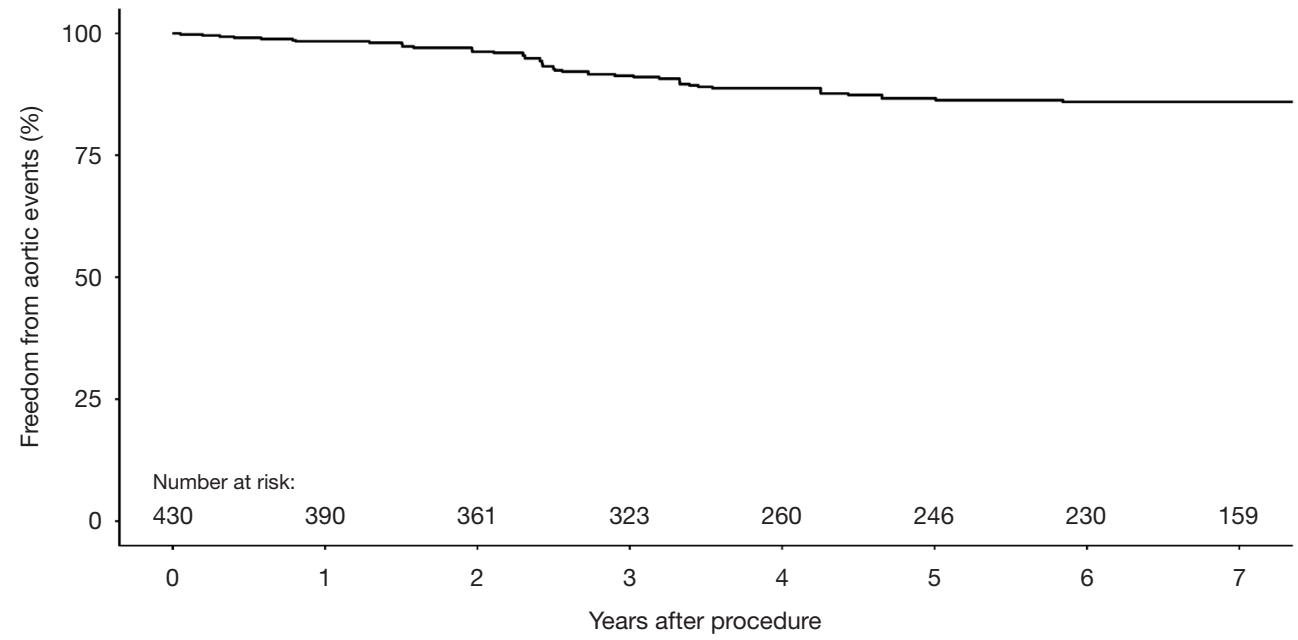

Figure 2 Freedom from aortic events.

\section{Discussion}

The present systematic review examined long-term outcomes of the FET technique. Aggregation of KaplanMeier curves found overall survival at 1-, 3-, and 5 -year were $89.6 \%, 85.2 \%$, and $82.0 \%$, respectively. In comparison, patients who received planned second-stage procedures after a classic elephant trunk had a 3-year survival rate of $75 \%$ (47). Indeed, the interval mortality between the first-stage and second-stage completion procedures ranges between $2-11 \%$ (48), with the latter operation greatly precluded by the use of the FET.
Furthermore, it has been shown that a patent false lumen in the descending aorta is a predictor for late mortality and need for reintervention due to aortic expansion $(49,50)$. In a meta-analysis of 11 cohort studies, residual patent false lumen was found to increase the risk of late mortality and aortic events in type A dissections by $71 \%$ and $179 \%$, respectively (50). The FET's ability to promote downstream remodelling and induce false lumen thrombosis has been well validated $(2,6)$, therefore providing an attractive option for management of such pathologies.

The need for reintervention after the FET procedure is not negligible. The ideal length of FET remains 


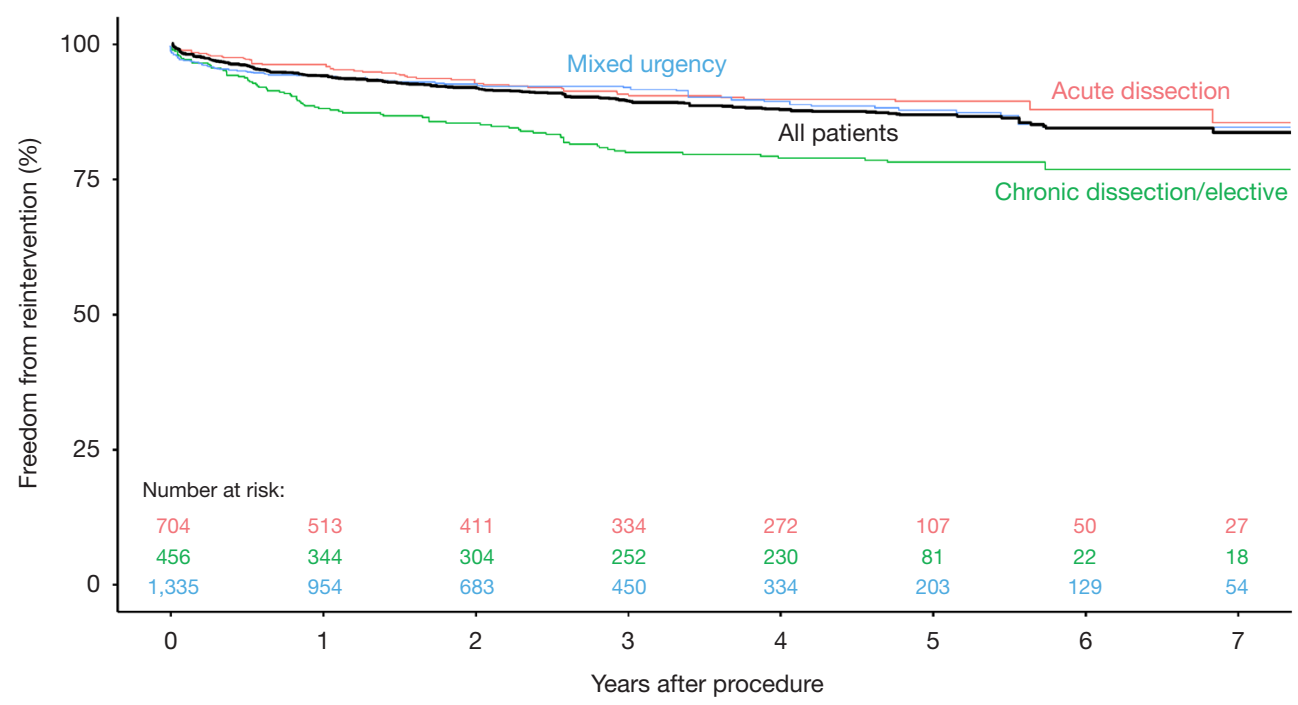

Figure 3 Freedom from reintervention.

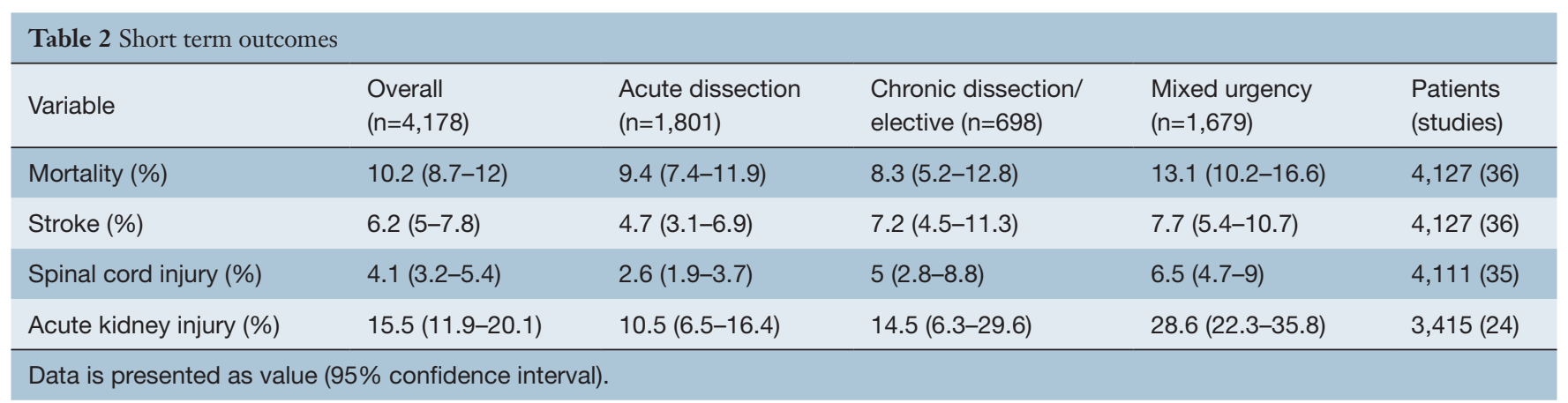

controversial, requiring careful balance between sufficient length to achieve adequate distal false lumen occlusion and minimizing occlusion of vascular collaterals that supply the spinal cord. As such, it is often not possible to provide full distal coverage of the aortic pathology due to fear of spinal cord ischemia, thereby necessitating a second-stage procedure despite the use of FETs $(43,51)$. However, it should be noted that the FET simplifies such reinterventions by providing a more appropriate landing zone for endovascular completion $(52,53)$. In the present review, freedom from reintervention at 1-, 3-, and 5-year was $93.9 \%, 89.3 \%$, and $86.8 \%$, respectively, reaffirming the need for close serial follow-up after the FET procedure.

There are several limitations to the present review that must be considered when interpreting these results. First, in order to attain sufficient statistical power and increase overall representativeness of the findings, this analysis included a heterogeneous cohort of patients, with varying comorbidities, pathologies, and surgical techniques. While subgroup classifications have been made based on clinical urgency, the assortment of surgical approaches, such as the extent of surgery, management of supra-aortic vessels (e.g., debranching procedures), neuroprotection strategies, and type and length of FETs is likely to have confounded results. Secondly, the volume of practice varied between hospitals, and particularly amongst geographic regions. Finally, the average length of follow-up is only 3.2 years, with limited data available beyond this period.

The present review demonstrates that survival after the FET procedure is favorable, though the need for reintervention still remains. Larger robust multiinstitutional registries are required to elucidate the precise role of the FET in managing complex multisegmental aortic pathologies. 


\section{Acknowledgments}

None.

\section{Footnote}

Conflicts of Interest: The authors have no conflicts of interest to declare.

Open Access Statement: This is an Open Access article distributed in accordance with the Creative Commons Attribution-NonCommercial-NoDerivs 4.0 International License (CC BY-NC-ND 4.0), which permits the noncommercial replication and distribution of the article with the strict proviso that no changes or edits are made and the original work is properly cited (including links to both the formal publication through the relevant DOI and the license). See: https://creativecommons.org/licenses/by-nc-nd/4.0/.

\section{References}

1. Shrestha M, Martens A, Kaufeld T, et al. Single-centre experience with the frozen elephant trunk technique in 251 patients over 15 years. Eur J Cardiothorac Surg 2017;52:858-66.

2. Berger T, Kreibich M, Morlock J, et al. True-lumen and false-lumen diameter changes in the downstream aorta after frozen elephant trunk implantation. Eur J Cardiothorac Surg 2018;54:375-81.

3. Koullias GJ, Wheatley GH, 3rd. State-of-the-art of hybrid procedures for the aortic arch: a meta-analysis. Ann Thorac Surg 2010;90:689-97.

4. Tian DH, Wan B, Di Eusanio M, et al. Short- and medium-term outcomes of the frozen elephant trunk technique in aortic arch surgery: A systematic review. Heart, Lung and Circulation 2015;24:e58-9.

5. Lin $\mathrm{HH}, \mathrm{Liao} \mathrm{SF}, \mathrm{Wu} \mathrm{CF}$, et al. Outcome of frozen elephant trunk technique for acute type A aortic dissection: as systematic review and meta-analysis. Medicine (Baltimore) 2015;94:e694.

6. Takagi H, Umemoto T. A Meta-Analysis of Total Arch Replacement with Frozen Elephant Trunk in Acute Type A Aortic Dissection. Vasc Endovascular Surg 2016;50:33-46.

7. Papakonstantinou NA, Antonopoulos CN, Baikoussis NG, et al. Frozen Elephant Trunk: An Alternative Surgical Weapon Against Extensive Thoracic Aorta Disease. A Three-Year Meta-Analysis. Heart Lung Circ
2019;28:213-22.

8. (IHE). IoHE. Quality Appraisal of Case Series Studies Checklist. Edmonton (AB): Institute of Health Economics, 2014.

9. Wan X, Wang W, Liu J, et al. Estimating the sample mean and standard deviation from the sample size, median, range and/or interquartile range. BMC Med Res Methodol 2014;14:135.

10. Guyot P, Ades A, Ouwens MJ, et al. Enhanced secondary analysis of survival data: reconstructing the data from published Kaplan-Meier survival curves. BMC Med Res Methodol 2012;12:9.

11. Saluja R, Cheng S, delos Santos KA, et al. Estimating hazard ratios from published Kaplan-Meier survival curves: A methods validation study. Res Synth Methods 2019;10:465-75.

12. Sun L, Qi R, Zhu J, et al. Total arch replacement combined with stented elephant trunk implantation: a new "standard" therapy for type a dissection involving repair of the aortic arch? Circulation 2011;123:971-8.

13. Zhao HP, Zhu JM, Ma WG, et al. Total arch replacement with stented elephant trunk technique for acute type B aortic dissection involving the aortic arch. Ann Thorac Surg 2012;93:1517-22.

14. Jakob H, Tsagakis K. International E-vita open registry. Ann Cardiothorac Surg 2013;2:296-9.

15. Shi E, Gu T, Yu Y, et al. Early and midterm outcomes of hemiarch replacement combined with stented elephant trunk in the management of acute DeBakey type I aortic dissection: comparison with total arch replacement. J Thorac Cardiovasc Surg 2014;148:2125-31.

16. Shi E, Gu T, Yu Y, et al. Simplified total arch repair with a stented graft for acute DeBakey type I dissection. J Thorac Cardiovasc Surg 2014;148:2147-54.

17. Zhang H, Lang X, Lu F, et al. Acute type A dissection without intimal tear in arch: proximal or extensive repair? J Thorac Cardiovasc Surg 2014;147:1251-5.

18. Katayama A, Uchida N, Katayama K, et al. The frozen elephant trunk technique for acute type A aortic dissection: results from 15 years of experience†. Eur J Cardiothorac Surg 2015;47:355-60; discussion 360.

19. Chen Q, Cheng F, Chen T, et al. Ascending aorta replacement combined with open placement of triplebranched stent graft and total arch replacement combined with stented elephant trunk implantation for treating type A aortic dissection. Int J Clin Exp Med 2016;9:16070-7.

20. Kobayashi M, Chaykovska L, van der Loo B, et al. Longterm results of simplified frozen elephant trunk technique 
in complicated acute type A aortic dissection: A casecontrol study. Vascular 2016;24:523-30.

21. Aalaei-Andabili SH, Scali S, Klodell C, et al. Outcomes of Antegrade Stent Graft Deployment During Hybrid Aortic Arch Repair. Ann Thorac Surg 2017;104:538-44.

22. Hu X, Wang Z, Ren Z, et al. Simplified total aortic arch replacement with an in situ stent graft fenestration technique for acute type A aortic dissection. J Vasc Surg 2017;66:711-7.

23. Yamane Y, Uchida N, Mochizuki S, et al. Early- and midterm aortic remodelling after the frozen elephant trunk technique for retrograde type A acute aortic dissection using the new Japanese J Graft open stent graft. Interact Cardiovasc Thorac Surg 2017;25:720-6.

24. Goebel N, Nagib R, Salehi-Gilani S, et al. One-stage hybrid aortic repair using the frozen elephant trunk in acute DeBakey type I aortic dissection. J Thorac Dis 2018;10:4195-203.

25. López Almodóvar LF, Lima Canadas P, Enriquez Puga A, et al. Single Low-Volume Center Experience with Frozen Elephant Trunk in Acute Type A Aortic Dissections. Aorta (Stamford) 2018;6:125-9.

26. Ma M, Feng X, Wang J, et al. Acute Type I aortic dissection: a propensity-matched comparison of elephant trunk and arch debranching repairs. Interact Cardiovasc Thorac Surg 2018;26:183-9.

27. Roselli EE, Idrees JJ, Bakaeen FG, et al. Evolution of Simplified Frozen Elephant Trunk Repair for Acute DeBakey Type I Dissection: Midterm Outcomes. Ann Thorac Surg 2018;105:749-55.

28. Kremer J, Preisner F, Dib B, et al. Aortic arch replacement with frozen elephant trunk technique - a single-center study. J Cardiothorac Surg 2019;14:147.

29. Lin Y, Ma WG, Zheng J, et al. Supra-aortic vessel reconstruction in total arch replacement for acute type A dissection: Comparison of en bloc and separate graft techniques. Asian J Surg 2019;42:482-7.

30. Qian SC, Ma WG, Pan XD, et al. Renal malperfusion affects operative mortality rather than late death following acute type A aortic dissection repair. Asian J Surg 2020;43:213-9.

31. Tochii M, Takami Y, Ishikawa H, et al. Aortic remodeling with frozen elephant trunk technique for Stanford type A aortic dissection using Japanese J-graft open stent graft. Heart Vessels 2019;34:307-15.

32. Yamamoto H, Kadohama T, Yamaura G, et al. Total arch repair with frozen elephant trunk using the "zone 0 arch repair" strategy for type A acute aortic dissection. J Thorac
Cardiovasc Surg 2019. [Epub ahead of print].

33. Flores J, Kunihara T, Shiiya N, et al. Extensive deployment of the stented elephant trunk is associated with an increased risk of spinal cord injury. J Thorac Cardiovasc Surg 2006;131:336-42.

34. Uchida N, Shibamura H, Katayama A, et al. Long-term results of the frozen elephant trunk technique for the extensive arteriosclerotic aneurysm. J Thorac Cardiovasc Surg 2010;139:913-7.

35. Nakamura K, Nagahama H, Nakamura E, et al. Predictors of early and late outcome after total arch replacement for atherosclerotic aortic arch aneurysm. Gen Thorac Cardiovasc Surg 2014;62:31-7.

36. Zhong YL, Qi RD, Ma WG, et al. Frozen elephant trunk with modified en bloc arch reconstruction and left subclavian transposition for chronic type A dissection. J Thorac Dis 2018;10:5376-83.

37. Leontyev S, Misfeld M, Daviewala P, et al. Early- and medium-term results after aortic arch replacement with frozen elephant trunk techniques-a single center study. Ann Cardiothorac Surg 2013;2:606-11.

38. Weiss G, Santer D, Dumfarth J, et al. Evaluation of the downstream aorta after frozen elephant trunk repair for aortic dissections in terms of diameter and false lumen status. Eur J Cardiothorac Surg 2016;49:118-24.

39. Mkalaluh S, Szczechowicz M, Mashhour A, et al. Total aortic arch replacement using elephant trunk or frozen elephant trunk technique: a case-control matching study. J Thorac Dis 2018;10:6192-200.

40. Tsagakis K, Wendt D, Dimitriou AM, et al. The frozen elephant trunk treatment is the operation of choice for all kinds of arch disease. J Cardiovasc Surg (Torino) 2018;59:540-6.

41. Alhussaini M, Abdelwahab A, Arnaoutakis GJ, et al. Neurologic Outcomes in Aortic Arch Repair With Frozen Elephant Trunk Versus 2-Stage Hybrid Repair. Ann Thorac Surg 2019;107:1775-81.

42. Chu MWA, Losenno KL, Dubois LA, et al. Early Clinical Outcomes of Hybrid Arch Frozen Elephant Trunk Repair With the Thoraflex Hybrid Graft. Ann Thorac Surg 2019;107:47-53.

43. Dinato FJ, Dias RR, Duncan JA, et al. The learning curve effect on outcomes with frozen elephant trunk technique for extensive thoracic aorta disease. J Card Surg 2019;34:796-802.

44. Kozlov BN, Panfilov DS, Saushkin VV, et al. Distal aortic remodelling after the standard and the elongated frozen elephant trunk procedure. Interact Cardiovasc Thorac 
Surg 2019;29:117-23.

45. Leone A, Di Marco L, Coppola G, et al. Open distal anastomosis in the frozen elephant trunk technique: initial experiences and preliminary results of arch zone 2 versus arch zone 3 dagger. Eur J Cardiothorac Surg 2019;56:564-71.

46. Zhang L, Yu C, Yang X, et al. Hybrid and frozen elephant trunk for total arch replacement in DeBakey type I dissection. J Thorac Cardiovasc Surg 2019;158:1285-92.

47. Svensson LG, Kim KH, Blackstone EH, et al. Elephant trunk procedure: newer indications and uses. Ann Thorac Surg 2004;78:109-16; discussion 116.

48. Safi HJ, Miller CC 3rd, Estrera AL, et al. Staged repair of extensive aortic aneurysms: morbidity and mortality in the elephant trunk technique. Circulation 2001;104:2938-42.

49. Fattouch K, Sampognaro R, Navarra E, et al. Longterm results after repair of type a acute aortic dissection according to false lumen patency. Ann Thorac Surg

Cite this article as: Tian DH, Ha H, Joshi Y, Yan TD. Longterm outcomes of the frozen elephant trunk procedure: a systematic review. Ann Cardiothorac Surg 2020;9(3):144-151. doi: 10.21037/acs.2020.03.08
2009;88:1244-50.

50. Li D, Ye L, He Y, et al. False lumen status in patients with acute aortic dissection: a systematic review and metaanalysis. J Am Heart Assoc 2016;5:e003172.

51. Uchida N, Kodama H, Katayama K, et al. Endovascular aortic repair as second-stage surgery after hybrid open arch repair by the frozen elephant trunk technique for extended thoracic aneurysm. Ann Thorac Cardiovasc Surg 2013;19:257-61.

52. Dias RR, Duncan JA, Vianna DS, et al. Surgical treatment of complex aneurysms and thoracic aortic dissections with the Frozen Elephant Trunk technique. Rev Bras Cir Cardiovasc 2015;30:205-10.

53. Ius F, Fleissner F, Pichlmaier $M$, et al. Total aortic arch replacement with the frozen elephant trunk technique: 10-year follow-up single-centre experience. Eur J Cardiothorac Surg 2013;44:949-57. 
Table S1 Study appraisal tool modified from Institute of Health Economics' Quality Appraisal Checklist for Case Series Studies (Canada). Each study was allocated a point for each criterion if it was deemed to have been met

Study appraisal schema

Was the hypothesis/aim/objective of the study clearly stated?

Was the study conducted prospectively?

Were the cases collected in more than one centre?

Were patients recruited consecutively?

Were the characteristics of the patients included in the study described?

Were the eligibility criteria (i.e., inclusion and exclusion criteria) for entry into the study clearly stated?

Did patients enter the study at a similar point in the disease?

Was the intervention of interest clearly described?

Were additional interventions (co-interventions) clearly described?

Were relevant outcome measures established a priori?

Were losses to follow-up reported?

Did the study provided estimates of random variability in the data analysis of relevant outcomes?

Were the adverse events reported?

Were the conclusions of the study supported by results?

Were both competing interests and sources of support for the study reported?

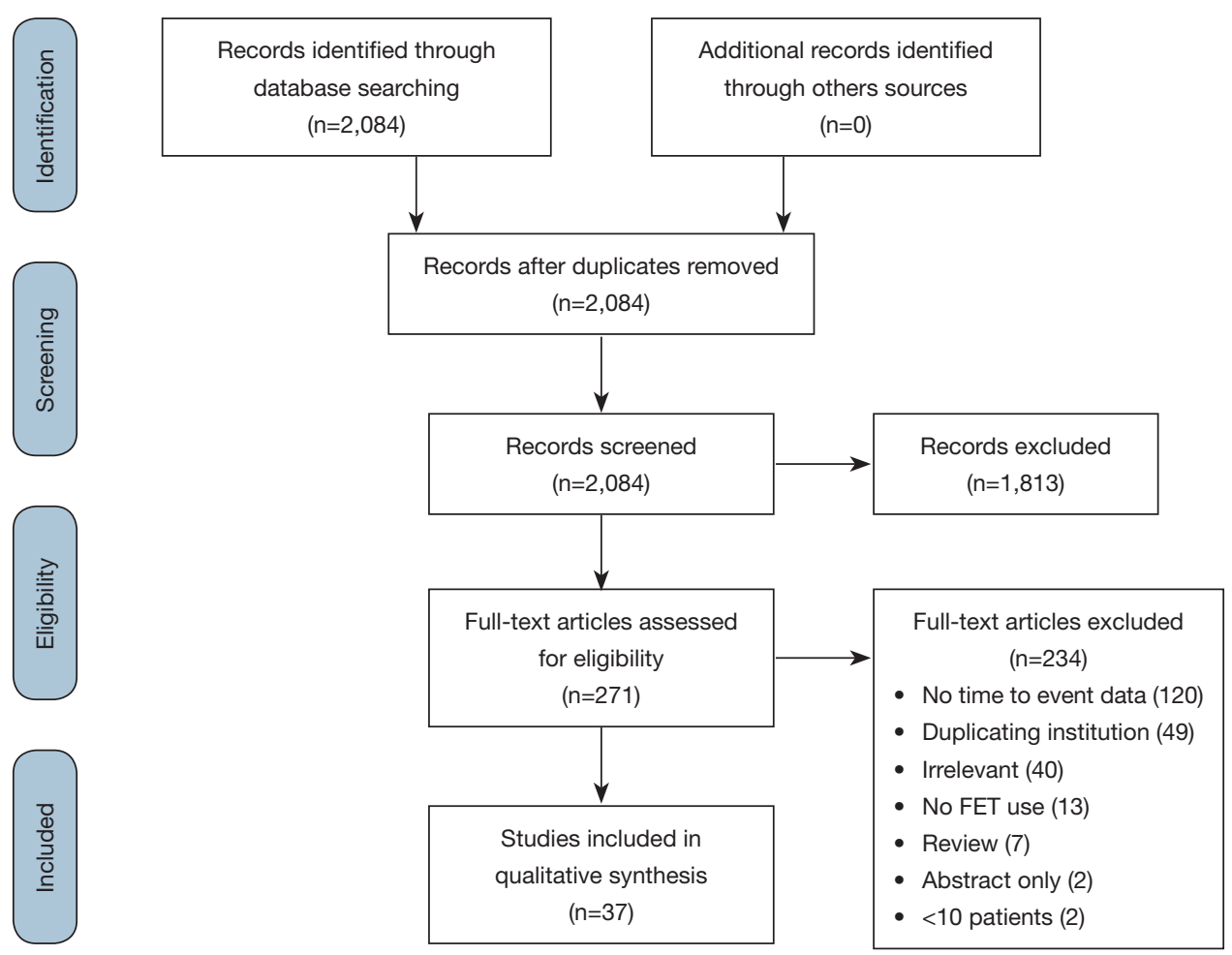

Figure S1 PRIMSA flow chart of literature search. 


\begin{tabular}{|c|c|c|c|c|c|c|c|c|}
\hline Author & $\begin{array}{l}\text { Year of } \\
\text { publication }\end{array}$ & Hospital & Country & Study period & Patients & Device name & $\begin{array}{l}\text { Follow-up } \\
\text { (months) }\end{array}$ & $\begin{array}{l}\text { Study } \\
\text { quality }\end{array}$ \\
\hline \multicolumn{9}{|l|}{ Acute/emergent } \\
\hline Sun & 2011 & Fuwai Hospital, Beijing Hospital, Beijing & China & 2003-2008 & 148 & Cronus & $42 \pm 18$ & Medium \\
\hline Zhao & 2012 & Beijing Anzhen Hospital, Beijing & China & 2006-2011 & 24 & Cronus & $37 \pm 21$ & Medium \\
\hline Jakob & 2013 & EVITA registry & International & 2005-2012 & 138 & E-Vita & NR & Low \\
\hline $\mathrm{Shi}^{\mathrm{a}}$ & 2014 & First Affiliated Hospital Medical University, Shenyang & China & 2006-2011 & 155 & Cronus & $43 \pm 18$ & Medium \\
\hline $\mathrm{Shi}^{\mathrm{b}}$ & 2014 & First Affiliated Hospital Medical University, Shenyang & China & 2010-2012 & 54 & Cronus & $24 \pm 9$ & Medium \\
\hline Zhang & 2014 & Changhai Hospital, Second Military Medical College, Shanghai & China & 2002-2010 & 88 & Cronus & $56 \pm 33$ & Medium \\
\hline Katayama & 2015 & Hiroshima-City Asa General Hospital, Hiroshima & Japan & $1997-2012$ & 120 & NR & $105 \pm 52$ & Medium \\
\hline Chen & 2016 & Tianjin Medical University, Tianjin & China & 2009-2014 & 33 & NR & NR & Low \\
\hline Kobayashi & 2016 & Zurich University Hospital, Zurich & Switzerland & 2001-2012 & 34 & GoreTAG, Talent, E-Vita & $74 \pm 45$ & Medium \\
\hline Aalaei-Andabili & 2017 & The University of Florida College of Medicine, Florida, USA & USA & 2010-2015 & 23 & GoreTAG, Valiant, TX2 & $17 \pm 17^{*}$ & High \\
\hline $\mathrm{Hu}$ & 2017 & Renmin Hospital of Wuhan University, Hubei & China & 2008-2015 & 106 & Cronus & $43 \pm 22$ & Medium \\
\hline Shrestha & 2017 & Hannover Medical School, Hannover & Germany & $2001-2016$ & 100 & $\begin{array}{l}\text { Chavan-Haverich, } \\
\text { E-Vita, Thoraflex }\end{array}$ & $54 \pm 57^{\star}$ & Medium \\
\hline Yamane & 2017 & Akane-Foundation Tsuchiya General Hospital, Hiroshima & Japan & 2008-2015 & 24 & JOSG & $39 \pm 22^{*}$ & Medium \\
\hline Berger & 2018 & University Heart Center Freiburg University, Freiburg & Germany & 2013-2017 & 31 & Thoraflex & $12 \pm 12$ & Medium \\
\hline Goebel & 2018 & Robert-Bosch Hospital, Stuttgart & Germany & 2009-2016 & 72 & E-Vita & $38 \pm 21$ & Medium \\
\hline Lopez Almodovar & 2018 & Virgen de la Salud Hospital, Toledo & Spain & $2011-2016$ & 12 & E-Vita & $36 \pm 29$ & Medium \\
\hline $\mathrm{Ma}$ & 2018 & Tongji Hospital, Wuhan & China & 2013-2015 & 132 & Cronus & $22 \pm 8^{*}$ & Medium \\
\hline Roselli & 2018 & Cleveland Clinic, Ohio, USA & USA & 2009-2016 & 72 & GoreTAG & $28 \pm 25$ & Low \\
\hline Kremer & 2019 & Heidelberg University Hospital, Heidelberg & Germany & $2006-2017$ & 34 & E-Vita, Thoraflex & NR & Medium \\
\hline Lin & 2019 & Beijing Anzhen Hospital, Beijing & China & 2013-2014 & 53 & Cronus & $52 \pm 19$ & Medium \\
\hline Qian & 2019 & Beijing Anzhen Hospital, Beijing & China & 2009-2012 & 218 & NR & $67 \pm 13$ & Medium \\
\hline Tochii & 2019 & Fujita Health University, Toyoake & Japan & $2005-2017$ & 22 & JOSG & $9 \pm 8$ & Low \\
\hline Yamamoto & 2019 & Akita University Hospital, Akita & Japan & 2014-2018 & 108 & Frozenix & NR & Medium \\
\hline \multicolumn{9}{|l|}{ Chronic/emergent } \\
\hline Flores & 2006 & Hokkaido University School of Medicine, Hokkaido & Japan & $1996-2004$ & 25 & $\begin{array}{l}\text { Gianturco stent/ } \\
\text { Hemashield Gold graft }\end{array}$ & $35 \pm 13$ & Low \\
\hline Uchida & 2010 & Hiroshima-City Asa General Hospital, Hiroshima & Japan & $1997-2008$ & 58 & NR & $54 \pm 37$ & Medium \\
\hline Sun & 2011 & FuWai Hospital, Beijing Hospital & China & $2003-2008$ & 143 & Cronus & $43 \pm 19$ & Medium \\
\hline Jakob & 2013 & EVITA registry & International & 2005-2012 & 142 & E-Vita & NR & Low \\
\hline Nakamura & 2014 & Miyazaki Prefectural Nobeoka Hospital, Nobeoka & Japan & $1998-2010$ & 51 & NR & 51 & Medium \\
\hline Aalaei-Andabili & 2017 & The University of Florida College of Medicine, Florida, USA & USA & 2010-2015 & 25 & GoreTAG, Valiant, TX2 & $17 \pm 17^{*}$ & High \\
\hline Shrestha & 2017 & Hannover Medical School, Hannover & Germany & $2001-2016$ & 151 & $\begin{array}{l}\text { Chavan-Haverich, } \\
\text { E-Vita, Thoraflex }\end{array}$ & $43 \pm 42^{*}$ & Medium \\
\hline Berger & 2018 & University Heart Center Freiburg University, Freiburg & Germany & 2013-2017 & 34 & Thoraflex & NR & Medium \\
\hline Zhong & 2018 & Beijing Anzhen Hospital, Beijing & China & 2010-2016 & 35 & Cronus & $49 \pm 22$ & Medium \\
\hline Kremer & 2019 & Heidelberg University Hospital, Heidelberg & Germany & $2006-2017$ & 34 & E-Vita, Thoraflex & NR & Medium \\
\hline \multicolumn{9}{|l|}{ Mixed } \\
\hline Leontyev & 2013 & Leipzig Heart Centre, Leipzig & Germany & $2006-2013$ & 51 & E-Vita & $41 \pm 5$ & Medium \\
\hline Weiss & 2016 & Hospital Hietzing, Vienna, Austria & Austria & 2005-2012 & 27 & E-Vita & $48 \pm 26$ & Medium \\
\hline Mkalaluh & 2018 & European Medical School Oldenburg-Groningen, Oldenburg & Germany & $2001-2017$ & 25 & E-Vita, Thoraflex & 26 & Low \\
\hline Tsagakis & 2018 & West German Heart Center Essen, Essen & Germany & $2005-2017$ & 286 & E-Vita & NR & Low \\
\hline Alhussaini & 2019 & Assuit University, Assiut & Egypt & 2003-2016 & 48 & TX2, Valiant & $31 \pm 32^{*}$ & Medium \\
\hline Chu & 2019 & 9 Canadian hospitals & $\begin{array}{l}\text { Multi- } \\
\text { Canadian }\end{array}$ & 2014-2017 & 40 & Thoraflex & $18 \pm 11$ & Medium \\
\hline Dinato & 2019 & University of Sao Paulo Medical School, Sao Paulo & Brazil & 2009-2018 & 79 & E-Vita & $17 \pm 20$ & Medium \\
\hline Kozlov & 2019 & Tomsk National Research Medical Centre, Tomsk & Russia & 2012-2018 & 26 & E-Vita & NR & Medium \\
\hline Leone & 2019 & Sant. Orsola-Malpighi Hospital, University of Bologna, Bologna & Italy & $2007-2018$ & 282 & E-Vita, Thoraflex & NR & Medium \\
\hline Zhang & 2019 & FuWai Hospital, Beijing Hospital & China & 2010-2016 & 815 & Cronus & NR & Medium \\
\hline
\end{tabular}

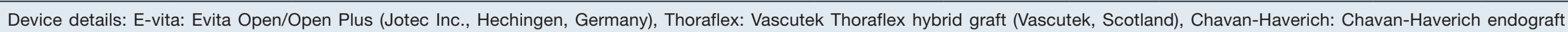

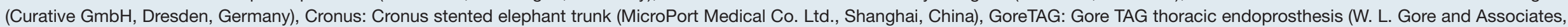

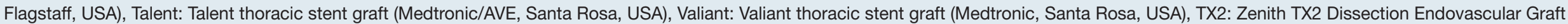

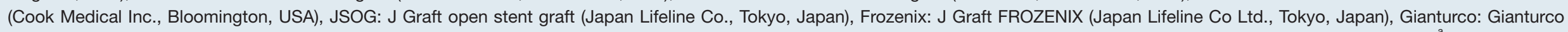

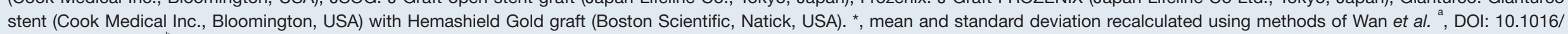
j.jtcvs.2013.10.058; ${ }^{\text {b }}$, DOI: 10.1016/j.jtcvs.2014.02.077. 


\begin{tabular}{|c|c|c|c|c|c|c|}
\hline Study & Age & Male & Hypertension & Diabetes & Renal dysfunction & Concomitant CABG \\
\hline \multicolumn{7}{|l|}{ Acute/emergent } \\
\hline Sun & $45 \pm 11$ & $126[85]$ & 107 [72] & $6[4]$ & 9 [6] & $17[11]$ \\
\hline Zhao & $41 \pm 1$ & 19 [79] & 15 [63] & $2[8]$ & NR & NR \\
\hline Jakob & $61^{\mathrm{M}}$ & NR & NR & NR & NR & NR \\
\hline $\mathrm{Shi}^{\mathrm{a}}$ & $56 \pm 10$ & $53[75]$ & $55[77]$ & $12[17]$ & $5[7]$ & $7[10]$ \\
\hline Shi $i^{a}$ & $54 \pm 12$ & 57 [68] & $67[80]$ & 19 [23] & $4[5]$ & $6[7]$ \\
\hline $\mathrm{Shi}^{\mathrm{b}}$ & $60 \pm 13$ & 37 [69] & $40[74]$ & $13[24]$ & $3[6]$ & $2[4]$ \\
\hline Zhang & $46 \pm 14$ & 74 [84] & 64 [73] & $4[5]$ & $3[3]$ & 8 [9] \\
\hline Katayama & $64 \pm 11$ & 64 [53] & NR & NR & $8[7]$ & NR \\
\hline Chen & $46 \pm 11$ & 18 [82] & $17[77]$ & $1[5]$ & NR & NR \\
\hline Chen & $54 \pm 11$ & $9[82]$ & 10 [91] & $0[0]$ & NR & NR \\
\hline Kobayashi & $60 \pm 11$ & $21[62]$ & $21[62]$ & $2[6]$ & NR & NR \\
\hline Aalaei-Andabili & $62 \pm 12$ & 14 [61] & $20[87]$ & 2 [9] & 2 [9] & $8[35]$ \\
\hline $\mathrm{Hu}$ & $51 \pm 12$ & 69 [65] & 77 [73] & NR & $4[4]$ & $10[9]$ \\
\hline Shrestha & NR & NR & NR & NR & NR & 13 [13] \\
\hline Yamane & $59 \pm 14$ & $14[58]$ & $14[58]$ & $0[0]$ & $3[13]$ & $3[13]$ \\
\hline Berger & $64 \pm 12$ & $24[77]$ & 25 [81] & $0[0]$ & $2[6]$ & $4[13]$ \\
\hline Goebel & $59 \pm 12$ & $55[76]$ & 68 [94] & $3[4]$ & $9[13]$ & $7[10]$ \\
\hline Lopez Almodovar & 57 & 10 [83] & 10 [83] & NR & NR & $0[0]$ \\
\hline $\mathrm{Ma}$ & $47 \pm 8$ & 108 [82] & 97 [73] & 31 [23] & $12[9]$ & $12[9]$ \\
\hline Roselli & $59 \pm 15$ & $51[71]$ & NR & NR & NR & $3[4]$ \\
\hline Kremer & $59 \pm 15$ & 28 [82] & 28 [82] & $1[3]$ & $2[6]$ & $2[6]$ \\
\hline Lin & $46 \pm 9$ & $41[77]$ & 44 [83] & NR & $4[8]$ & 5 [9] \\
\hline Qian & $48 \pm 11$ & $170[78]$ & $153[70]$ & 5 [2] & 48 [22] & $20[9]$ \\
\hline Tochii & $60 \pm 36^{*}$ & 18 [82] & NR & NR & NR & $1[5]$ \\
\hline Yamamoto & $67 \pm 12$ & $50[46]$ & 32 [30] & $4[4]$ & $4[4]$ & $5[5]$ \\
\hline Subtotal & 45 [45-45] & 73 [68-78] & 74 [68-80] & 7 [4-12] & 7 [5-11] & 10 [8-12] \\
\hline \multicolumn{7}{|l|}{ Chronic/elective } \\
\hline Flores & $73 \pm 7$ & 19 [76] & $21[84]$ & $2[8]$ & 3 [12] & 3 [12] \\
\hline Uchida & 74 & 52 [90] & 53 [91] & 15 [26] & 13 [22] & $15[26]$ \\
\hline Sun & $45 \pm 10$ & 112 [78] & $100[70]$ & $5[3]$ & 3 [2] & $8[6]$ \\
\hline Jakob & $60 \mathrm{M}$ & NR & NR & NR & NR & NR \\
\hline Nakamura & NR & NR & NR & NR & NR & NR \\
\hline Aalaei-Andabili & $66 \pm 10$ & 17 [68] & 19 [76] & $4[16]$ & $3[12]$ & $4[16]$ \\
\hline Shrestha & NR & NR & NR & NR & NR & $23[28]$ \\
\hline Shrestha & NR & NR & NR & NR & NR & $8[12]$ \\
\hline Berger & $58 \pm 12$ & 24 [71] & 31 [91] & $1[3]$ & $7[21]$ & 1 [3] \\
\hline Zhong & $49 \pm 10$ & 29 [83] & 27 [77] & $4[11]$ & 3 [9] & $2[6]$ \\
\hline Kremer & $65 \pm 11$ & 20 [59] & 29 [85] & $2[6]$ & 3 [9] & 7 [21] \\
\hline Subtotal & 55 [54-56] & 76 [68-83] & 82 [74-88] & 9 [4-18] & $11[6-19]$ & 14 [8-21] \\
\hline \multicolumn{7}{|l|}{ Mixed } \\
\hline Leontyev & $69 \pm 10$ & 27 [53] & 27 [53] & 9 [18] & NR & 10 [20] \\
\hline Weiss & $56 \pm 12$ & $21[78]$ & 17 [63] & $6[22]$ & $3[11]$ & $0[0]$ \\
\hline Mkalaluh & $67 \pm 9^{*}$ & $14[56]$ & 18 [72] & NR & NR & $5[20]$ \\
\hline Tsagakis & $59 \pm 11$ & 199 [70] & NR & $22[8]$ & 59 [21] & NR \\
\hline Alhussaini & $64 \pm 11$ & 31 [65] & 39 [81] & $6[13]$ & NR & $13[27]$ \\
\hline Chu & $66 \pm 14$ & 22 [55] & 32 [80] & $5[13]$ & $1[3]$ & $6[15]$ \\
\hline Dinato & $58 \pm 13$ & 50 [63] & 70 [89] & $12[15]$ & 14 [18] & $6[8]$ \\
\hline Kozlov & $54 \pm 11$ & $10[67]$ & $12[80]$ & $1[7]$ & NR & NR \\
\hline Kozlov & $52 \pm 4$ & 7 [64] & $9[82]$ & $1[9]$ & NR & NR \\
\hline Leone & $54 \pm 48^{*}$ & 233 [83] & 237 [84] & $11[4]$ & $13[5]$ & $26[9]$ \\
\hline Zhang & $47 \pm 10$ & 637 [78] & 594 [73] & 19 [2] & $40[5]$ & 82 [10] \\
\hline Subtotal & 52 [52-53] & 68 [61-75] & 76 [69-82] & 9 [5-15] & 9 [4-18] & 13 [9-19] \\
\hline Overall & 57 [54-60] & 72 [68-75] & 76 [72-80] & 8 [6-12] & 8 [6-11] & 11 [9-14] \\
\hline
\end{tabular}

Data is presented as number of patients [\%], or mean \pm standard deviation. Aggregated subtotal and overall values are presented with $95 \%$ confidence intervals, using random-effects meta-analysis of proportions or means. ${ }^{*}$, mean and standard deviation recalculated using methods of Wan et al. ${ }^{\text {a }}$, DOI: 10.1016/j.jtcvs.2013.10.058; ${ }^{\text {, }}$, DOI: 10.1016/j.jtcvs.2014.02.077. M, median. 
Table S4 Intraoperative characteristics

\begin{tabular}{|c|c|c|c|c|c|}
\hline Study & CPB time (mins) & Cross-clamp time (mins) & HCA time (mins) & ACP time (mins) & Lowest temp $\left({ }^{\circ} \mathrm{C}\right)$ \\
\hline \multicolumn{6}{|l|}{ Acute/emergent } \\
\hline Sun & $197 \pm 47$ & $107 \pm 27$ & NR & $24 \pm 9$ & $18-22$ \\
\hline Zhao & $168 \pm 41$ & $87 \pm 24$ & NR & $21 \pm 5$ & 20 \\
\hline Jakob & $241 \pm 75$ & NR & $140 \pm 54$ & $68 \pm 30$ & NR \\
\hline $\mathrm{Shi}^{\mathrm{a}}$ & $104 \pm 21$ & $76 \pm 16$ & $31 \pm 5$ & $31 \pm 5$ & $23 \pm 2$ \\
\hline $\mathrm{Shi}^{\mathrm{a}}$ & $165 \pm 20$ & $109 \pm 18$ & $29 \pm 4$ & $55 \pm 6$ & $24 \pm 1$ \\
\hline $\mathrm{Shi}^{\mathrm{b}}$ & $96 \pm 18$ & $76 \pm 16$ & $29 \pm 3$ & $27 \pm 2$ & $23 \pm 2$ \\
\hline Zhang & $182 \pm 34$ & $113 \pm 26$ & $35 \pm 12$ & NR & $20-28$ \\
\hline Katayama & $173 \pm 42$ & $109 \pm 23$ & NR & $72 \pm 20$ & 28 \\
\hline Chen & $142 \pm 36$ & $103 \pm 28$ & $41 \pm 17$ & NR & $18-22$ \\
\hline Chen & $135 \pm 33$ & $103 \pm 29$ & $38 \pm 9$ & NR & $18-22$ \\
\hline Kobayashi & NR & NR & NR & NR & NR \\
\hline Aalaei-Andabili & $253 \pm 114$ & NR & $33 \pm 32$ & NR & $19 \pm 4$ \\
\hline $\mathrm{Hu}$ & $163 \pm 68$ & $93 \pm 22$ & $23 \pm 6$ & NR & 20 \\
\hline Shrestha & $254 \pm 64^{\star}$ & $119 \pm 67^{*}$ & NR & $84 \pm 30^{*}$ & $20-25$ \\
\hline Yamane & $255 \pm 57$ & $126 \pm 39$ & $49 \pm 145$ & $120 \pm 46$ & $27 \pm 1$ \\
\hline Berger & $228 \pm 52$ & $135 \pm 52$ & NR & $95 \pm 44$ & $24 \pm 1$ \\
\hline Goebel & $226 \pm 46^{*}$ & $157 \pm 35^{\star}$ & $71 \pm 17^{*}$ & NR & 28 \\
\hline Lopez Almodovar & $235 \pm 43$ & $171 \pm 33$ & $75 \pm 20$ & $96 \pm 23$ & 25 \\
\hline $\mathrm{Ma}$ & $243 \pm 66$ & $122 \pm 26$ & $27 \pm 9$ & NR & $22 \pm 2$ \\
\hline Roselli & NR & NR & $33 \pm 22$ & NR & 20 \\
\hline Kremer & $252 \pm 74$ & $148 \pm 35$ & $62 \pm 37$ & NR & $23 \pm 4$ \\
\hline Lin & $199 \pm 62$ & $108 \pm 39$ & NR & $27 \pm 9$ & 25 \\
\hline Qian & $199 \pm 59$ & $111 \pm 36$ & NR & $29 \pm 14$ & NR \\
\hline Tochii & $296 \pm 80$ & $183 \pm 49$ & $88 \pm 24$ & $206 \pm 57$ & NR \\
\hline Yamamoto & $214 \pm 65^{\star}$ & $133 \pm 47^{*}$ & $53 \pm 14^{*}$ & $108 \pm 37$ & $23 \pm 2$ \\
\hline Subtotal & 199 [177-221] & 117 [107-126] & 48 [43-53] & 65 [57-74] & 24 [24-24] \\
\hline \multicolumn{6}{|l|}{ Chronic/elective } \\
\hline Flores & NR & NR & NR & NR & 22 \\
\hline Uchida & $148 \pm 34$ & NR & NR & $72 \pm 12$ & 28 \\
\hline Sun & $182 \pm 38$ & $102 \pm 28$ & NR & $24 \pm 6$ & $18-22$ \\
\hline Jakob & NR & NR & NR & NR & NR \\
\hline Nakamura & NR & NR & NR & NR & $20-25$ \\
\hline Aalaei-Andabili & $270 \pm 59$ & NR & $29 \pm 25$ & NR & $19 \pm 3$ \\
\hline Shrestha & $203 \pm 63^{*}$ & $97 \pm 47^{*}$ & NR & $68 \pm 29^{*}$ & $20-25$ \\
\hline Shrestha & $237 \pm 68^{*}$ & $202 \pm 68^{\star}$ & NR & $81 \pm 31^{*}$ & $20-25$ \\
\hline Berger & $219 \pm 58$ & $116 \pm 51$ & NR & $76 \pm 37$ & $25 \pm 3$ \\
\hline Zhong & $176 \pm 47$ & $89 \pm 30$ & NR & $29 \pm 6$ & 25 \\
\hline Kremer & $189 \pm 48$ & $116 \pm 35$ & $55 \pm 37$ & NR & $24 \pm 2$ \\
\hline Subtotal & 202 [179-225] & 120 [97-143] & 42 [16-67] & 58 [40-76] & 23 [23-24] \\
\hline \multicolumn{6}{|l|}{ Mixed } \\
\hline Leontyev & $213 \pm 66$ & $98 \pm 38$ & $50 \pm 14$ & $47 \pm 14$ & $26 \pm 2$ \\
\hline Weiss & $204 \pm 46$ & $95 \pm 34$ & $4 \pm 2$ & $54 \pm 12$ & 26 \\
\hline Mkalaluh & $229 \pm 53$ & $100 \pm 58$ & $42 \pm 33$ & NR & $25 \pm 2^{*}$ \\
\hline Tsagakis & NR & NR & NR & NR & NR \\
\hline Alhussaini & $268 \pm 81$ & NR & $31 \pm 28$ & NR & NR \\
\hline Chu & $246 \pm 78$ & $155 \pm 72$ & $47 \pm 21$ & NR & NR \\
\hline Dinato & $155 \pm 31$ & $119 \pm 33$ & NR & $60 \pm 13$ & 25 \\
\hline Kozlov & $237 \pm 82^{*}$ & $161 \pm 40^{*}$ & $63 \pm 28^{*}$ & $47 \pm 22^{*}$ & NR \\
\hline Kozlov & $186 \pm 27^{\star}$ & $148 \pm 61^{*}$ & $55 \pm 10^{*}$ & $39 \pm 22^{*}$ & NR \\
\hline Leone & $329 \pm 400^{\star}$ & $157 \pm 191^{*}$ & $60 \pm 65^{*}$ & $138 \pm 197^{\star}$ & 25 \\
\hline Zhang & $196 \pm 64$ & $101 \pm 29$ & $23 \pm 8$ & NR & $20-25$ \\
\hline Subtotal & 222 [200-243] & 123 [110-136] & $41[31-51]$ & 59 [49-68] & 26 [25-26] \\
\hline Overall & 206 [191-220] & 118 [112-125] & $46[41-51]$ & 63 [56-69] & 23 [23-24] \\
\hline
\end{tabular}

Data is presented as number of patients [\%] or mean \pm standard deviation. Aggregated subtotal and overall values are presented with $95 \%$ confidence intervals, using random-effects meta-analysis of proportions or means. Temperatures also presented as ranges as per individual studies. *, mean and standard deviation recalculated using methods of Wan et al. ${ }^{\text {a }}$, DOI: 10.1016/j.jtcvs.2013.10.058; ${ }^{\text {b }}$, DOI: 10.1016/j.jtcvs.2014.02.077. 
Table S5 Clinical outcomes

\begin{tabular}{|c|c|c|c|c|c|}
\hline Study & Mortality & Stroke & Spinal cord injury & Acute kidney injury & ICU stay [days] \\
\hline \multicolumn{6}{|l|}{ Acute/emergent } \\
\hline Sun & 7 [5] & $4[3]$ & $3[2]$ & $1[1]$ & NR \\
\hline Zhao & $1[4]$ & $0[0]$ & $0[0]$ & NR & NR \\
\hline Jakob & $22[16]$ & $10[7]$ & $6[4]$ & NR & NR \\
\hline $\mathrm{Shi}^{\mathrm{a}}$ & $3[4]$ & $0[0]$ & $0[0]$ & $4[6]$ & NR \\
\hline $\mathrm{Shi}^{\mathrm{a}}$ & $5[6]$ & $0[0]$ & $0[0]$ & $7[8]$ & NR \\
\hline $\mathrm{Shi}^{\mathrm{b}}$ & $2[4]$ & $0[0]$ & $0[0]$ & $4[7]$ & NR \\
\hline Zhang & $5[6]$ & 2 [2] & $0[0]$ & 2 [2] & NR \\
\hline Katayama & $7[6]$ & $4[3]$ & 2 [2] & $4[3]$ & NR \\
\hline Chen & $6[27]$ & $0[0]$ & NR & NR & $10 \pm 11$ \\
\hline Chen & $3[27]$ & $1[9]$ & NR & NR & $12 \pm 12$ \\
\hline Kobayashi & 5 [15] & $2[6]$ & NR & NR & NR \\
\hline Aalaei-Andabili & $4[17]$ & $2[9]$ & $1[4]$ & $6[26]$ & $11 \pm 6^{\star}$ \\
\hline $\mathrm{Hu}$ & $8[8]$ & $0[0]$ & $0[0]$ & NR & $3 \pm 2$ \\
\hline Shrestha & $11[11]$ & $18[18]$ & $1[1]$ & $21[21]$ & $6 \pm 5^{*}$ \\
\hline Yamane & $2[8]$ & $1[4]$ & $0[0]$ & NR & NR \\
\hline Berger & $2[6]$ & $4[13]$ & $0[0]$ & NR & NR \\
\hline Goebel & $11[15]$ & $2[3]$ & $3[4]$ & 18 [25] & $6 \pm 5^{*}$ \\
\hline Lopez Almodovar & $2[17]$ & $1[8]$ & $0[0]$ & NR & NR \\
\hline $\mathrm{Ma}$ & $19[14]$ & $7[5]$ & $0[0]$ & 20 [15] & $8 \pm 4$ \\
\hline Roselli & $3[4]$ & $3[4]$ & $3[4]$ & $2[3]$ & NR \\
\hline Kremer & 5 [15] & $3[9]$ & $3[9]$ & $23[68]$ & $9 \pm 9$ \\
\hline Lin & $3[6]$ & $1[2]$ & $0[0]$ & $5[9]$ & $4 \pm 4$ \\
\hline Qian & $25[11]$ & $2[1]$ & 5 [2] & $35[16]$ & $3 \pm 3$ \\
\hline Tochii & $0[0]$ & $4[18]$ & $0[0]$ & $2[9]$ & $11 \pm 12$ \\
\hline Yamamoto & $3[3]$ & $4[4]$ & $0[0]$ & $6[6]$ & NR \\
\hline Subtotal & 9.4 [7.4-11.9] & $4.7[3.1-6.9]$ & $2.6[1.9-3.7]$ & $10.5[6.5-16.4]$ & $7[5-8]$ \\
\hline \multicolumn{6}{|l|}{ Chronic/elective } \\
\hline Flores & $3[12]$ & $4[16]$ & $6[24]$ & NR & NR \\
\hline Uchida & $1[2]$ & $2[3]$ & $2[3]$ & $1[2]$ & NR \\
\hline Sun & $2[1]$ & 3 [2] & $4[3]$ & $2[1]$ & NR \\
\hline Jakob & $20[14]$ & $7[5]$ & $13[9]$ & NR & NR \\
\hline Nakamura & NR & NR & $2[4]$ & NR & NR \\
\hline Aalaei-Andabili & $4[16]$ & $1[4]$ & $1[4]$ & $6[24]$ & $13 \pm 9^{*}$ \\
\hline Shrestha & $9[11]$ & $11[13]$ & $3[4]$ & $16[20]$ & $5 \pm 5^{*}$ \\
\hline Shrestha & $3[4]$ & 8 [12] & $0[0]$ & 15 [22] & $7 \pm 8^{*}$ \\
\hline Berger & $2[6]$ & $2[6]$ & $0[0]$ & NR & NR \\
\hline Zhong & $2[6]$ & $0[0]$ & $0[0]$ & NR & NR \\
\hline Kremer & 5 [15] & $4[12]$ & $2[6]$ & $16[47]$ & $9 \pm 9$ \\
\hline Subtotal & 8.3 [5.2-12.8] & 7.2 [4.5-11.3] & 5 [2.8-8.8] & 14.5 [6.3-29.6] & 8 [5-11] \\
\hline \multicolumn{6}{|l|}{ Mixed } \\
\hline Leontyev & $4[8]$ & $6[12]$ & $10[20]$ & 13 [25] & NR \\
\hline Weiss & $2[7]$ & $3[11]$ & $2[7]$ & $8[30]$ & $4 \pm 11^{*}$ \\
\hline Mkalaluh & $5[20]$ & $6[24]$ & $1[4]$ & $6[24]$ & $11 \pm 11^{*}$ \\
\hline Tsagakis & $32[11]$ & $16[6]$ & $14[5]$ & 91 [32] & NR \\
\hline Alhussaini & $9[19]$ & $3[6]$ & $2[4]$ & NR & NR \\
\hline Chu & $2[5]$ & $3[8]$ & $2[5]$ & $1[3]$ & $2 \pm 2^{*}$ \\
\hline Dinato & $16[20]$ & $4[5]$ & $2[3]$ & $38[48]$ & NR \\
\hline Kozlov & $0[0]$ & $1[7]$ & $0[0]$ & NR & NR \\
\hline Kozlov & $0[0]$ & $0[0]$ & $0[0]$ & NR & NR \\
\hline Leone & $48[17]$ & $25[9]$ & $17[6]$ & 55 [20] & $1 \pm 3^{*}$ \\
\hline Zhang & $87[11]$ & $36[4]$ & $56[7]$ & 263 [32] & $3 \pm 3$ \\
\hline Subtotal & $13.1[10.2-16.6]$ & $7.7[5.4-10.7]$ & $6.5[4.7-9]$ & 28.6 [22.3-35.8] & $3[1-4]$ \\
\hline Overall & 10.2 [8.7-12] & $6.2[5-7.8]$ & $4.1[3.2-5.4]$ & 15.5 [11.9-20.1] & $6[5-7]$ \\
\hline
\end{tabular}

Data is presented as number of patients [\%] or mean \pm standard deviation. Aggregated subtotal and overall values are presented with $95 \%$ confidence intervals, using random-effects meta-analysis of proportions or means. ${ }^{*}$, mean and standard deviation recalculated using methods of Wan et al. ${ }^{a}$, DOI: 10.1016/j.jtcvs.2013.10.058; ${ }^{b}$, DOI: 10.1016/j.jtcvs.2014.02.077. 